\title{
Perfil epidemiológico da coinfecção TB/HIV em um município prioritário da Amazônia ocidental
}

\author{
Perfil epidemiológico de la coinfección Tuberculosis/VIH en un municipio \\ prioritario de la Amazonía occidental
}

Epidemiological profile of Tuberculosis/HIV coinfection in a prioritary city of Western Amazon

Recebido: 16 abr 2021

Revisado: 25 jun 2021

Aceito: 5 jul 2021

Autor de correspondência: Amanda Silveira Mariano asilveiramariano@gmail.com

Como citar: Mariano AS

Magnabosco GT, Orfão NH.

Perfil epidemiológico da coinfecção TB/HIV em um município prioritário da Amazônia ocidental. J Manag Prim Health Care. 2021;13:e08. https://doi.org/10.14295/jmphc.v13. 1131

Conflito de interesses:

Os autores declaram não haver nenhum interesse profissional ou pessoal que possa gerar conflito de interesses em relação a este manuscrito.

Copyright: Este é um artigo de acesso aberto, distribuído sob os termos da Licença Creative Commons (CC-BYNC). Esta licença permite que outros distribuam, remixem, adaptem e criem a partir do seu trabalho, mesmo para fins comerciais, desde que the atribuam o devido crédito pela criação original.

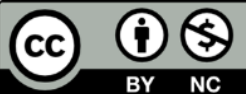

Amanda Silveira MARIANO(1) Gabriela Tavares MAGNABOSCO(2) Nathalia Halax ORFÃO(1)

\footnotetext{
(1) Universidade Federal de Rondônia - UNIR, Porto Velho, RO, Brasil.

(2) Universidade Estadual de Maringá - UEM, Maringá, PR, Brasil.
}

\section{Resumo}

A infecção pelo vírus da imunodeficiência humana (HIV) aumenta o risco de desenvolver tuberculose (TB), favorecendo a coinfecção. Este estudo teve como objetivo analisar os casos de coinfecção TB/HIV em Porto Velho-RO, no período de 2014 a 2018. Trata-se de um estudo descritivo, do tipo transversal e abordagem quantitativa, realizado a partir do levantamento das variáveis sociodemográficas e clínicas no Sistema de Informação de Agravos de Notificação e analisados por meio de estatística descritiva, após atendidos os preceitos éticos. Das 276 pessoas com coinfecção TB/HIV, a idade média foi de 38,3 anos $(\mathrm{dp}= \pm 11,28)$, a maioria era do sexo masculino $(73,9 \%)$, raça/cor autodeclarada parda (79,3\%), escolaridade entre 5 e 8 anos (33,3\%), presença de aids (98,2\%), alcoolismo (21\%), uso de drogas ilícitas $(14,5 \%)$, tabagismo $(9 \%)$, privados de liberdade $(5,4 \%)$, caso novo (62\%), forma pulmonar (62\%), radiografia de tórax suspeita (79,3\%), baciloscopia de escarro negativa $(44,6)$, cultura de escarro $(86,6 \%)$ e Teste Molecular Rápido para TB (71\%) não realizados, e teste de sensibilidade $(84,4 \%)$ e baciloscopias de escarro para controle mensal em branco/ignorado. Os casos foram notificados, em sua maioria, no serviço de referência terciária (67\%), porém tratados no Serviço de Assistência Especializada (68,5\%), tinham entre 1 e 5 contatos identificados (74\%), porém nenhum examinado (74,3\%), não realizaram tratamento diretamente observado (TDO) $(84,4 \%)$, com tempo médio de tratamento de $147,2$ dias ( $\mathrm{dp}= \pm 88,15)$, baixa taxa de cura $(42 \%)$ e elevada para abandono $(34,4 \%)$. Os resultados apontam para importantes fragilidades na realização dos exames diagnósticos e no acompanhamento dos casos coinfectados, bem como vigilância dos contatos, além da implementação restrita do TDO e baixo tempo médio de tratamento, contribuindo, portanto, para os desfechos insatisfatórios, conforme encontrado neste estudo.

Descritores: Infecções por HIV; Perfil de Saúde; Tuberculose. 


\title{
Resumen
}

La infección por el Virus de la Inmunodeficiencia Humana - VIH aumenta el riesgo de desarrollar tuberculosis (TB), favoreciendo la coinfección. Este estudio tuvo como objetivo analizar los casos de coinfección TB/VIH en Porto Velho-RO, de 2014 a 2018. Se trata de un estudio descriptivo, transversal con enfoque cuantitativo, realizado a partir de la encuesta de sociodemográficos y clínicas en el Sistema de Información de Enfermedades Notificables y analizadas mediante estadística descriptiva, previo cumplimiento de los preceptos éticos. De las 276 personas con coinfección TB/VIH, la edad media fue de 38,3 años ( $\mathrm{dt}= \pm 11,28)$, la mayoría eran hombres $(73,9 \%)$, autodeclarados de raza/color marrón (79,3\% ), educación entre 5 y 8 años (33,3\%), presencia de SIDA (98,2\%), alcoholismo (21\%), uso de drogas ilícitas (14,5\%), tabaquismo (9\%), eran privados libertad (5,4\%), caso nuevo (62\%), forma pulmonar (62\%), sospecha de radiografía de tórax (79,3\%), baciloscopia de esputo negativa $(44,6)$, cultivo de esputo $(86,6 \%)$ y Prueba Molecular Rápida para TB $(71 \%)$ no realizada, y prueba de sensibilidad $(84,4 \%)$ y microscopía de frotis de esputo para control mensual en blanco/ignorado. La mayoría de los casos se notificaron en el servicio de derivación terciaria (67\%), pero atendidos en el Servicio de Asistencia Especializada (68,5\%), tenían entre 1 y 5 contactos identificados (74\%), pero ninguno examinado (74,3\%), no se sometieron a tratamiento directamente observado (TDO) $(84,4 \%)$, con un tiempo de tratamiento promedio de 147,2 días ( $D E= \pm 88,15$ ), tasa de curación baja (42\%) y alta de abandono (34,4\%). Los resultados apuntan a importantes debilidades en la realización de pruebas diagnósticas y en el seguimiento de casos coinfectados, así como en la vigilancia de contactos, además de la implementación restringida del ODD y el bajo tiempo promedio de tratamiento, contribuyendo así a los resultados insatisfactorios encontrados en este estudio.

Palabras-claves: Infecciones por VIH; Perfil de Salud; Tuberculosis.

\begin{abstract}
The infection by the Human Immunodeficiency Virus - HIV increases the risk of developing tuberculosis (TB), favoring co-infection. This study aimed to analyze the TB/HIV co-infection cases in Porto Velho-RO, from 2014 to 2018. It is a descriptive, cross-sectional study with a quantitative approach, based on the survey of sociodemographic and clinics variables in the Notifiable Diseases Information System and analyzed by descriptive statistics, after complying with the ethical precepts. Of the 276 people with TB/HIV co-infection, the mean age was 38.3 years ( $\mathrm{sd}= \pm 11.28$ ), mostly male (73.9\%), brown color (79.3\% ), educational level between 5 and 8 years (33.3\%), with AIDS (98.2\%), alcoholism (21\%), use of illicit drugs (14.5\%), smoking (9\%), were deprived of liberty (5.4\%), new cases (62\%), pulmonary form (62\%), suspected chest X-ray (79.3\%), negative sputum smear microscopy (44.6), sputum culture (86,6\%) and Rapid Molecular Test for TB (71\%) not performed, and sensitivity test $(84.4 \%)$ and sputum smear microscopy for monthly control in blank/ignored. Most cases were reported at the tertiary referral service (67\%), but treated at the Specialized Assistance Service (68.5\%), had between 1 and 5 contacts identified (74\%), but none examined (74.3\%), did not perform Directly Observed Treatment (DOT) (84.4\%), with an average treatment time of 147.2 days (SD $= \pm 88.15)$, low cure rate $(42 \%)$ and high for abandonment (34.4\%). The results point to important weaknesses in the performance of diagnostic tests and in the monitoring of co-infected cases, as well as surveillance of contacts, in addition to the restricted implementation of DOT and low average treatment time, thus contributing to the unsatisfactory outcomes found in this study.
\end{abstract}

Keywords: HIV Infections; Health Profile; Tuberculosis.

\section{Introdução}

A tuberculose (TB) é uma doença que, apesar de tratável e curável, representa grande ameaça à saúde pública, pois atinge cerca de 10 milhões de pessoas todos os anos, sendo uma das principais causas de morte em todo o mundo. ${ }^{1} \mathrm{~A}$ infecção pelo vírus da imunodeficiência humana (HIV) eleva o risco de desenvolver TB em 28 vezes, $^{2}$ sendo que, em 2019, 8,6\% dos casos novos eram coinfectados TB/HIV no Brasil. ${ }^{3}$ 
A infecção por TB causa grande impacto na mortalidade em pessoas vivendo com HIV (PVHIV), sendo a principal causa de morte nesta população, demandando, portanto, medidas de prevenção e controle da TB. Para isso, o Ministério da Saúde - MS preconiza que todo indivíduo diagnosticado com TB seja testado para o HIV, notificado no Sistema de Informação de Agravos de Notificação - SINAN, tratado, monitorado e avaliado durante todo o período de tratamento, incluindo a realização de exames periódicos e controle de contatos, ${ }^{4}$ através do fornecimento gratuito das medicações pelo Sistema Único de Saúde - SUS.

Sabe-se, no entanto, que a implementação dessas medidas enfrenta diversos desafios estruturais, como o déficit de profissionais e dificuldade de acesso aos serviços por parte dos usuários; organizacionais, através da fragmentação da atenção e falta de comunicação entre os serviços; e, por fim, políticos e socioeconômicos, pelo caráter social e estigmatizante tanto da TB quanto do HIV, que atinge em maior número aqueles em situação de vulnerabilidade e invisibiliza esses indivíduos, afetando a resolutividade dos serviços de saúde para o enfrentamento da TB. ${ }^{5,6}$

Nesse sentido, destaca-se a importância de reconhecer as características predominantes nos casos de coinfecção TB/HIV, bem como as formas de manifestação clínica da TB nesses indivíduos e o manejo dos casos desde a entrada no serviço de saúde até o seu encerramento, com o intuito de compreender e avaliar a dinâmica adotada no controle e tratamento da TB entre as PVHIV para promover qualidade de vida dessa população.

Portanto, este estudo buscou analisar os casos de coinfecção TB/HIV em Porto VelhoRO, no período de 2014 a 2018.

\section{Metodologia}

Trata-se de um estudo descritivo, do tipo transversal e abordagem quantitativa desenvolvido em Porto Velho, capital do estado de Rondônia, com uma população estimada, em 2019, de 529.544 habitantes. $^{7}$ Conta com 37 Unidades de Saúde da Família - USF e 17 Unidades Básicas de Saúde - UBS distribuídos em unidades terrestres e ribeirinhas, bem como uma unidade móvel fluvial, que constituem porta de entrada preferencial para os casos de TB e/ou HIV, além de um Serviço de Assistência Especializada - SAE, onde são atendidos os casos de coinfecção TB/HIV, um Centro de Especialidades Médicas para o acompanhamento da forma clínica extrapulmonar e TB infantil, um hospital de referência para doenças infectocontagiosas para os casos de internação e, por fim, apoio laboratorial para exames diagnósticos e de controle. ${ }^{8}$

A população deste estudo foi constituída por todos os registros dos casos coinfectados TB/HIV, notificados no SINAN, no período entre 2014 a 2018. Considerou-se 
como critério de inclusão, os indivíduos com idade igual ou superior a 18 anos e que residissem em Porto Velho. E como exclusão, aqueles cuja variável situação de encerramento estivesse em branco ou ignorado.

Os dados foram coletados por meio de um levantamento das variáveis sociodemográficas (idade, raça/cor, escolaridade, doença e agravos associados, e população especial); clínicas (tipo de entrada, forma clínica, exames diagnósticos (radiografia de tórax, baciloscopia de escarro, cultura de escarro, Teste Molecular Rápido para a TB - TMR-TB e teste de sensibilidade), HIV e de controle mensal (baciloscopia de escarro); e do tratamento (controle de contatos, regime de tratamento diretamente observado (TDO), tempo de tratamento e situação de encerramento) no SINAN e armazenados no Programa Microsoft Excel e analisados por meio de estatística descritiva no software Statistica 13.4, da TIBCO.

Este estudo faz parte do projeto matriz intitulado "Avaliação da situação epidemiológica da tuberculose no município de Porto Velho - RO", o qual foi aprovado pelo Comitê de Ética em Pesquisa - CEP da Universidade Federal de Rondônia - UNIR, conforme parecer 2.399.327.

\section{Resultado}

Foram notificados no SINAN, entre 2014 e 2018, 3.804 casos de TB no estado de Rondônia, sendo que 11,3\% (430) desses casos foram positivos para o HIV, ainda que $16 \%$ não tenham realizado o exame e $1 \%$ ainda estivesse em andamento. Dentre as pessoas com coinfecção TB/HIV, 64,9\% (279) eram residentes da capital do estado, sendo que, destas, uma foi excluída por apresentar idade inferior a 18 anos e dois por apresentarem a variável situação de encerramento em branco; totalizando 276 casos para este estudo, cuja média de idade foi de 38,3 anos ( $d p= \pm 11,28)$, sendo a mínima de 18 e a máxima 74 anos.

Quanto ao perfil sociodemográfico, a maioria era do sexo masculino (73,9\%), raça/cor parda (79,3\%), com escolaridade entre 5 e 8 anos (33,3\%), dentre as doenças e agravos associados, verificou-se aids (98,2\%), alcoolismo (21,0\%), uso de drogas ilícitas (14,5\%), tabagismo (9\%), diabetes (1,8\%), doença mental $(1,1 \%)$ e outros agravos $(5,1 \%)$, e se constituía como população privada de liberdade (PPL) (5,4\%), população em situação de rua (PSR) $(2,2 \%)$, profissional de saúde $(0,8 \%)$ e imigrante $(0,8 \%)$ (Tabela 1$)$.

Tabela 1. Distribuição do perfil sociodemográfico dos casos coinfectados TB/HIV notificados no SINAN, Porto Velho-RO, no período entre 2014 e 2018

\begin{tabular}{cccc}
\hline \hline Sexo & Variáveis & N & $\%$ \\
\hline & & & \\
\hline & Masculino & 204 & 73,9 \\
\hline & Feminino & 72 & 26,1 \\
\hline
\end{tabular}


ISSN 2179-6750

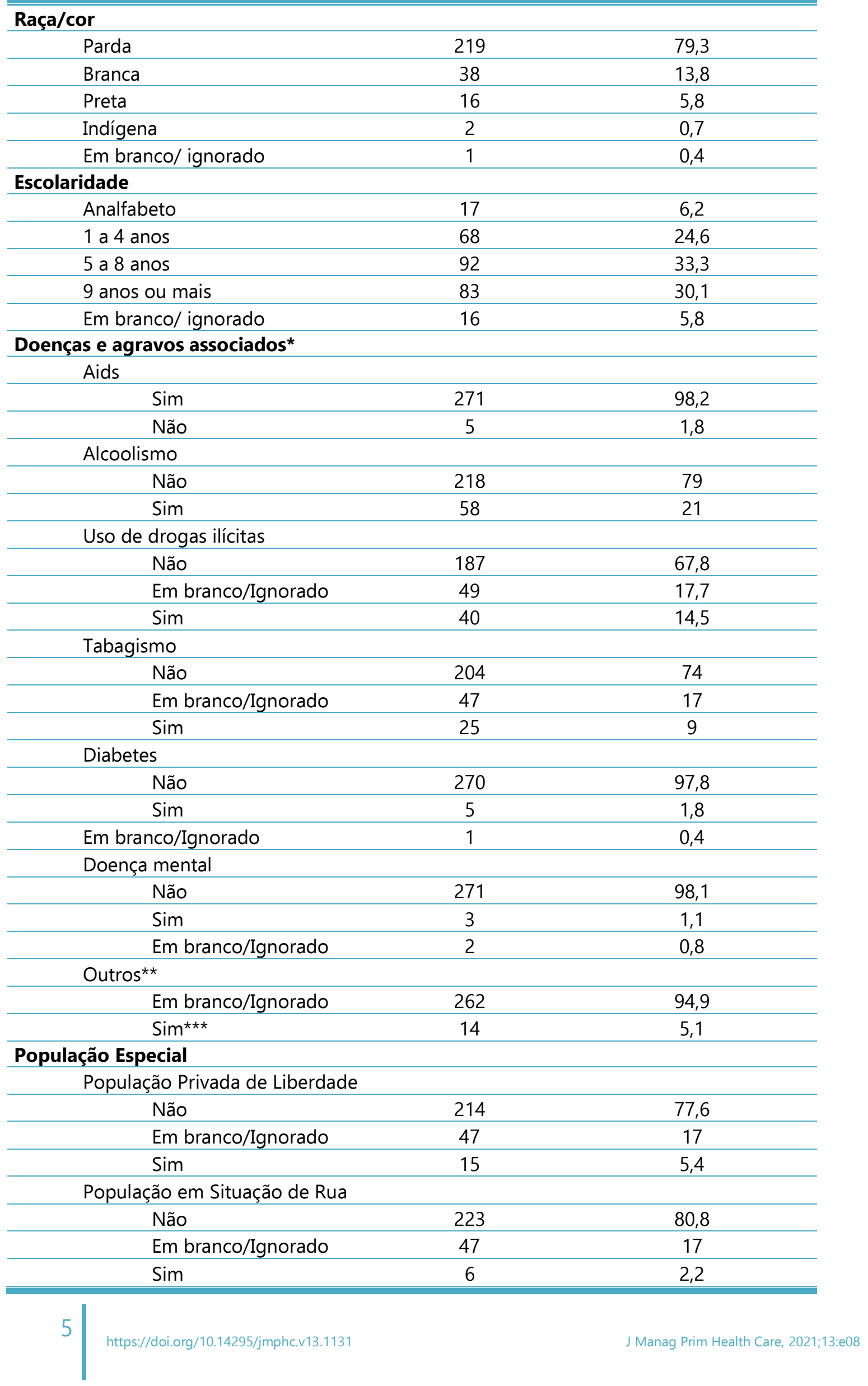


ISSN 2179-6750

\begin{tabular}{ccc}
\hline \hline Profissional de Saúde & & \\
\hline Não & 227 & 82,8 \\
\hline Em branco/Ignorado & 47 & 17 \\
\hline Sim & 2 & 0,8 \\
\hline Imigrante & & \\
\hline Não & 227 & 82,2 \\
\hline Em branco/Ignorado & 47 & 17 \\
\hline Sim & 2 & 0,8 \\
\hline \hline
\end{tabular}

Fonte: Elaborado pelos autores (2020).

Notas: *Um mesmo indivíduo pode apresentar mais de uma doença ou agravo associado simultaneamente. Não foram considerados nesta variável indivíduos que não possuem doenças e agravos associados ou não possuem registro desse dado no sistema. **Inclui hipertensão arterial sistólica, câncer de intestino, infecção por citomegalovírus, pericardite, pangastrite, hipotireoidismo, hepatite $B$, sífilis e bronquite asmática. ${ }^{* \star *} 6$ indivíduos pertencentes à categoria "uso de drogas ilícitas" foram inseridos na variável "outros" com a descrição no preenchimento como "maconha" (1) e "drogadição" (5).

Quanto ao perfil clínico, a maioria era caso novo (62\%), forma clínica pulmonar (62\%), radiografia de tórax suspeita de TB (79,3\%), baciloscopia de escarro negativa (44,6\%), cultura de escarro (86,6\%) e TMR-TB (71\%) não realizados, e teste de sensibilidade em branco/ignorado (84,8\%). Quanto aos exames de controle mensal, verificou-se elevados percentuais de preenchimento em branco. A notificação dos casos aconteceu majoritariamente na referência terciária (67\%) e realizaram o tratamento no SAE (68,5\%). Na maioria dos casos, foram identificados entre 1 e 5 contatos (74\%), nenhum contato foi examinado (74,3\%) e não realizaram TDO (84,4\%) (Tabela 2$)$.

Tabela 2. Distribuição do perfil clínico e do tratamento dos casos coinfectados TB/HIV notificados no SINAN, Porto Velho-RO, no período entre 2014 e 2018

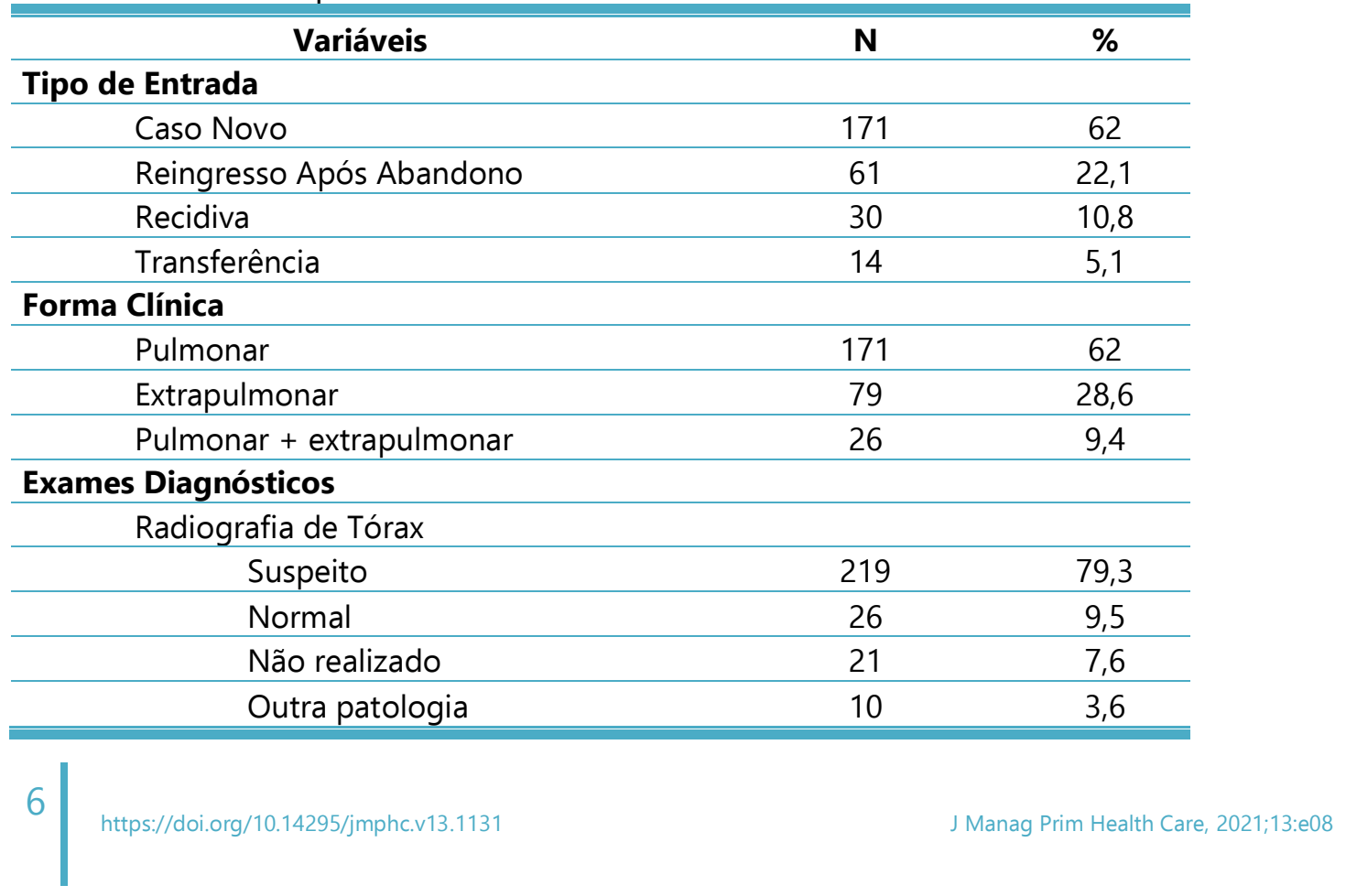


ISSN 2179-6750

Baciloscopia de Escarro

$\begin{array}{lcc}\text { Negativo } & 123 & 44,6 \\ \text { Positivo } & 80 & 29 \\ \text { Não realizado } & 69 & 25 \\ \text { Não se aplica } & 4 & 1,4\end{array}$

Cultura de escarro

Não realizado $\quad 239 \quad 86,6$

Em andamento $\quad 17 \quad 6,2$

Positivo $10 \quad 3,6$

$\begin{array}{lll}\text { Negativo } & 10 & 3,6\end{array}$

TMR-TB*

Não realizado $\quad 154 \quad 71$

Não detectável $\quad 35 \quad 16,1$

$\begin{array}{lll}\text { Detectável sensível à } & 24 & 11,1 \\ \text { Rifampicina } & \end{array}$

Inconclusivo $\quad 4 \quad 1,8$

Teste de Sensibilidade

\begin{tabular}{lcc} 
Em branco/Ignorado & 234 & 84,8 \\
Não Realizado & 36 & 13 \\
\hline Sensível & 3 & 1,1 \\
Em andamento & 3 & 1,1
\end{tabular}

Exames de Controle Mensal**

Baciloscopia do $1^{\circ}$ mês

$\begin{array}{lcc}\text { Em branco/Ignorado } & 104 & 52,8 \\ \text { Não realizado } & 74 & 37,6 \\ \text { Não se aplica } & 10 & 5,1 \\ \text { Negativo } & 7 & 3,5 \\ \text { Positivo } & 2 & 1\end{array}$

Baciloscopia do $2^{\circ}$ mês

\begin{tabular}{lcc} 
Em branco/Ignorado & 110 & 55,9 \\
Não realizado & 69 & 35 \\
\hline Não se aplica & 10 & 5,1 \\
\hline Negativo & 7 & 3,5 \\
\hline Positivo & 1 & 0,5
\end{tabular}

Baciloscopia do $3^{\circ}$ mês

\begin{tabular}{lcc} 
Em branco/Ignorado & 121 & 61,4 \\
\hline Não realizado & 59 & 30 \\
\hline Não se aplica & 10 & 5,1 \\
Negativo & 6 & 3 \\
\hline Positivo & 1 & 0,5 \\
\hline copia do $4^{\circ}$ mês & & \\
\hline Em branco/Ignorado & 128 & 65 \\
\hline Não realizado & 52 & 26,4 \\
\hline Não se aplica & 10 & 5,1 \\
Negativo & 7 & 3,5 \\
\hline
\end{tabular}


ISSN 2179-6750

\begin{tabular}{|c|c|c|}
\hline \multicolumn{3}{|l|}{ Baciloscopia do $5^{\circ}$ mês } \\
\hline Em branco/Ignorado & 133 & 67,5 \\
\hline Não realizado & 48 & 24,4 \\
\hline Não se aplica & 10 & 5,1 \\
\hline Negativo & 5 & 2,5 \\
\hline Positivo & 1 & 0,5 \\
\hline \multicolumn{3}{|l|}{ Baciloscopia do $6^{\circ}$ mês } \\
\hline Em branco/Ignorado & 135 & 69,3 \\
\hline Não realizado & 42 & 21,5 \\
\hline Não se aplica & 10 & 5,1 \\
\hline Negativo & 7 & 3,6 \\
\hline Positivo & 1 & 0,5 \\
\hline \multicolumn{3}{|l|}{ Unidade de Notificação } \\
\hline Referência Terciária & 185 & 67 \\
\hline SAE & 69 & 25 \\
\hline USF/UBS & 16 & 5,8 \\
\hline Complexo Penitenciário & 6 & 2,2 \\
\hline \multicolumn{3}{|l|}{ Tratamento } \\
\hline \multicolumn{3}{|l|}{ Contatos identificados } \\
\hline Nenhum contato & 37 & 13,4 \\
\hline Entre 1 e 5 contatos & 204 & 74 \\
\hline 6 ou mais contatos & 35 & 12,6 \\
\hline \multicolumn{3}{|l|}{ Contatos examinados } \\
\hline Nenhum contato & 205 & 74,3 \\
\hline Entre 1 e 5 contatos & 20 & 7,2 \\
\hline Em branco/ ignorado & 51 & 18,5 \\
\hline \multicolumn{3}{|l|}{ Regime de TDO } \\
\hline Não & 233 & 84,4 \\
\hline Sim & 1 & 0,4 \\
\hline Em branco/ ignorado & 42 & 15,2 \\
\hline \multicolumn{3}{|l|}{ Unidade de Tratamento } \\
\hline SAE & 189 & 68,5 \\
\hline Referência Terciária & 54 & 19,6 \\
\hline USF/UBS & 21 & 7,6 \\
\hline Complexo Penitenciário & 10 & 3,6 \\
\hline CEM & 2 & 0,7 \\
\hline
\end{tabular}

Fonte: Elaborado pelos autores (2020).

Notas: TMR-TB - Teste Molecular Rápido para TB; TDO - Tratamento Diretamente Observado; USF - Unidade de Saúde da Família; UBS - Unidade Básica de Saúde; SAE - Serviço de Assistência Especializada; CEM - Centro de Especialidades Médicas. *Considerando a implantação do TMR-TB em 2015, foi excluído o ano de 2014 para esta variável $(\mathrm{N}=217)$. ${ }^{* *}$ Foram considerados apenas os casos de TB pulmonar e TB pulmonar + extrapulmonar.

O tempo médio de tratamento da TB dos casos de coinfecção TB/HIV foi de 147,2 dias $(\mathrm{dp}= \pm 88,15)$, sendo o máximo de 385 dias. Ressalta-se que um caso foi excluído em 
relação ao tempo, considerando que iniciou o tratamento antes do diagnóstico, de acordo com os registros no SINAN.

Quanto a situação de encerramento, verificou-se baixa taxa de cura (42\%) ainda que tenha ocorrido prioritariamente acima de 180 dias (88,8\%), além de elevado percentual de abandono (34,4\%), principalmente entre 91 a 180 dias (45,3\%), quando também ocorreu outros desfechos, tais como o óbito por TB (100\%), óbitos por outras causas (35,3\%), mudança de diagnóstico (66,7\%), TB drogarresistente (TB-DR) (100\%) e falência (100\%). Quanto às transferências, a maioria ocorreu antes do $30^{\circ}$ dia de tratamento (52\%) e a mudança de esquema entre o $30^{\circ}$ e o $90^{\circ}$ dia (60\%) (Tabela 3 ).

Tabela 3. Distribuição da situação de encerramento, de acordo com o tempo dos casos de coinfecção TB/HIV segundo os registros no SINAN, Porto Velho-RO, no período entre 2014 e 2018

\begin{tabular}{|c|c|c|c|c|c|c|c|c|c|c|}
\hline \multirow[t]{3}{*}{ Situação de encerramento } & \multicolumn{10}{|c|}{ Tempo de tratamento (dias) } \\
\hline & \multicolumn{2}{|c|}{$<30$} & \multicolumn{2}{|c|}{$\begin{array}{c}\text { Entre } 30 \text { e } \\
90 \text { dias }\end{array}$} & \multicolumn{2}{|c|}{$\begin{array}{c}\text { Entre } 91 \text { e } 180 \\
\text { dias }\end{array}$} & \multicolumn{2}{|c|}{$\begin{array}{c}\text { Acima de } 180 \\
\text { dias }\end{array}$} & \multicolumn{2}{|c|}{ TOTAL } \\
\hline & $\mathrm{n}$ & $\%$ & $\mathrm{n}$ & $\%$ & $\mathrm{n}$ & $\%$ & $N$ & $\%$ & $\mathrm{n}$ & $\%$ \\
\hline Cura & 5 & 4,3 & 4 & 3,4 & 4 & 3,4 & 103 & 88,8 & 116 & 42,0 \\
\hline Abandono & 6 & 6,3 & 22 & 23,2 & 43 & 45,3 & 24 & 25,3 & 95 & 34,4 \\
\hline Óbito por TB & - & - & - & - & 2 & 100,0 & - & - & 2 & 0,7 \\
\hline Óbito por outras causas & 4 & 23,5 & 5 & 29,4 & 6 & 35,3 & 2 & 11,8 & 17 & 6,2 \\
\hline Transferência & 13 & 52,0 & 8 & 32,0 & 2 & 8,0 & 2 & 8,0 & 25 & 9,1 \\
\hline Mudança diagnóstico & 1 & 33,3 & - & - & 2 & 66,7 & - & - & 3 & 1,1 \\
\hline $\mathrm{TB}-\mathrm{DR}{ }^{*}$ & 0 & 0,0 & - & - & 2 & 100,0 & - & - & 2 & 0,7 \\
\hline Mudança de Esquema & 6 & 40,0 & 9 & 60,0 & - & - & - & - & 15 & 5,4 \\
\hline Falência & - & - & - & - & 1 & 100,0 & - & - & 1 & 0,4 \\
\hline
\end{tabular}

Fonte: Elaborado pelos autores (2020)

Notas: *Tuberculose Drogarresistente.

\section{Discussão}

Diante dos achados encontrados neste estudo, observa-se evidências de possível subnotificação dos casos de coinfecção TB/HIV no município estudado, considerando que $16 \%$ das pessoas com TB não foram testadas para o HIV, ainda que o MS recomende que todos as pessoas com de TB sejam testadas para o HIV. ${ }^{4}$ Isso significa que, uma vez que a infecção por HIV representa um grande fator de risco para o adoecimento por TB, mais de 600 pessoas poderiam estar potencialmente infectadas pelo vírus, desconhecerem o fato e permanecerem transmitindo para outras pessoas, evidenciando falha no manejo dos casos. Além disso, ficou evidente a importância da qualidade de retroalimentação do sistema de informação, uma vez que $1 \%$ estava com o resultado do exame em andamento. 


\section{Perfil sociodemográfico}

ISSN 2179-6750

O perfil das pessoas com coinfecção TB/HIV no período estudado retrata uma população majoritariamente masculina, de raça/cor parda e baixa escolaridade, o que está alinhado com o perfil encontrado em outros estados do país. ${ }^{9-12}$ Tais características estão relacionadas a diversos fatores, dentre eles os culturais e comportamentais, uma vez que a construção da masculinidade na sociedade desencoraja o autocuidado e favorece a adoção de comportamentos de risco. ${ }^{13}$ A raça/cor parda, devido ao advento da miscigenação, compõe cerca de $70,2 \%$ da população da Região Norte. ${ }^{14}$

A baixa escolaridade também representa um desafio para a adoção de práticas preventivas, compreensão dos problemas de saúde e participação do sujeito no seu processo de cuidado, pois prejudica a capacidade de entender, interpretar e aplicar as informações recebidas, levando a menor adesão a tratamentos e cuidados, menor utilização dos serviços e, consequentemente, declínio das condições de saúde. ${ }^{15}$ Nesse sentido, cabe à equipe a adoção de estratégias para superação deste desafio, utilizando métodos que estimulem melhor comunicação entre profissional e usuário durante as consultas, de forma a superar barreiras socioculturais e promover melhor entendimento do indivíduo quanto ao seu problema de saúde e método terapêutico.

Além disso, a escolaridade e a situação socioeconômica são determinantes que costumam caminhar juntos, na qual é possível inferir que o baixo grau de instrução prevalente nos indivíduos coinfectados pode estar relacionada ao fato de que a TB é, sobretudo, uma doença social que emerge da iniquidade na distribuição de renda, dado que é um agravo que atinge com maior impacto aqueles em situação de pobreza, vulnerabilidade e exclusão social. ${ }^{16}$

Da população que compõe o estudo, 98,2\% têm aids, ou seja, estão na fase clínica mais avançada da infecção por HIV, cujo potencial imunodebilitante favorece o surgimento de infecções oportunistas e agrava quadros clínicos de patologias pré-existentes. Além da coinfecção TB/HIV, que por si só ameaça à saúde desses indivíduos, já que a TB é principal causa de morte em PVHIV, alguns apresentam ainda outras comorbidades adicionais e comportamentos de risco, como doenças crônicas, infecções sexualmente transmissíveis, uso de drogas ilícitas, tabagismo e alcoolismo, aumentando a vulnerabilidade dos usuários a desfechos desfavoráveis, uma vez que prejudicam a evolução clínica da TB e demonstraram-se fortes determinantes para o abandono do tratamento, impossibilitando o processo de cura e contribuindo para a resistência medicamentosa. ${ }^{6}$

No caso de associação com outras comorbidades crônicas, como a diabetes, alguns sintomas já causados pela coinfecção TB/HIV podem ser intensificados, como inapetência, perda de peso e febre, agravando a situação clínica do paciente, além de dificultar o 
tratamento devido ao acúmulo de alterações fisiológicas causados pelas patologias. ${ }^{17}$ Assim, deve-se atentar não somente à coinfecção TB/HIV de forma isolada, mas investigar cuidadosamente cada caso para a possibilidade de agravos associados, propiciando um manejo integrado para as diferentes comorbidades, de forma a minimizar os problemas causados pelas doenças e auxiliar no controle e tratamento.

Das populações especiais, as mais prevalentes no estudo foram a PPL e a PSR. No que tange a PPL, o cenário de confinamento e aglomeração e a dificuldade na realização de medidas de controle de infecções e as características sociais e econômicas peculiares, tornam essa população mais propensa a se infectar e desenvolver TB, com risco de adoecimento 28 vezes superior à população geral. ${ }^{2} \mathrm{O}$ risco de se infectar pelo HIV também é maior, tanto pelo compartilhamento de objetos perfurocortantes para uso de drogas injetáveis quanto pela prática sexual desprotegida, especialmente em casos de violência. ${ }^{18}$

Dessa forma, o ambiente penitenciário demonstra-se oportuno para proliferação de ambas as doenças, favorecendo, portanto, a coinfecção TB/HIV, condição que requer maior articulação entre os serviços de saúde para manejo e controle, o que pode se tornar um grande desafio para os sistemas de saúde e prisional, especialmente porque, ainda que a notificação possa acontecer em outros serviços devido à complexidade nos sinais e sintomas necessitando de internação, por exemplo, o tratamento da TB será realizado dentro das unidades prisionais, conforme ocorreu neste estudo.

Além dos desafios estruturais que favorecem a proliferação de doenças infectocontagiosas no complexo prisional, como a superlotação, estudos apontam para outras limitações enfrentados pelo sistema carcerário, especialmente no controle da TB. De acordo com Ferreira et al., ${ }^{19}$ apesar do grande número de casos notificados de TB na PPL, existem ainda desafios nas condutas básicas para identificação e controle dos casos, uma vez que as ações de saúde não são priorizadas nesses ambientes, como a baixa realização da busca ativa, exames diagnósticos e falta de acompanhamento do tratamento, que fragilizam o combate à TB e contribui para a vulnerabilidade desta população.

No caso da PSR, existe grande associação às condições e situações de vida que ampliam os riscos em saúde. Em estudo realizado por Rossetto et al. ${ }^{20}$ identificou-se que, muitas vezes, o indivíduo sequer sabe quando adquiriu a TB ou quando se infectou por HIV, a coinfecção só era identificada já em estágio avançado devido a internação simultaneamente de ambas as doenças, demonstrando fragilidade no vínculo dos sujeitos com os serviços de saúde. Tal aspecto é especialmente grave nessas situações, uma vez que PSR vivem em condições de extrema vulnerabilidade e devem ser compreendidas dentro de sua complexidade. 


\section{Perfil clínico}

ISSN 2179-6750

Quanto ao perfil clínico dos casos, a maioria era caso novo e forma pulmonar, o que também está de acordo com o que é apresentado em outras literaturas. ${ }^{11,10}$ No entanto, destaca-se o elevado percentual de reingresso após abandono (22,1\%), que, reflete igualmente o abandono (34,4\%) e aponta para elevados casos de interrupção do tratamento por tempo igual ou superior a 30 dias, seja por desconhecimento da importância de manter o tratamento mesmo com a melhora dos sinais e sintomas, dificuldades na adesão ao tratamento, falta de conhecimento, estigma da doença, dentre outros fatores, evidenciando fragilidade no vínculo com os serviços de saúde ou nas orientações fornecidas pelos profissionais.

Ainda que a TB extrapulmonar não seja considerada um fator de risco em relação à transmissão da doença, esta pode não ser detectada através da baciloscopia de escarro e gera manifestações clínicas que podem ser confundidas com outras patologias, tornando o seu diagnóstico mais complexo. Além disso, pode afetar diversos sistemas e causar o comprometimento dos mesmos, comumente associada a quadros clínicos graves da doença, principalmente em pacientes imunocomprometidos, ${ }^{21}$ como é o caso das PVHIV.

Sobre a dificuldade na adesão do tratamento, que constitui importante desafio em relação ao abandono, pode acontecer por vários fatores, como a quantidade de medicamentos, tempo de tratamento, tamanho da medicação que torna sua ingestão desconfortável e efeitos adversos. No entanto, vale ressaltar que alguns aspectos podem ser atenuados através de ações de educação permanente em saúde para orientação e realização adequada do acompanhamento do tratamento, fortalecimento de vínculo entre profissionais-usuário-família-comunidade e adoção de estratégias de acompanhamento direto das pessoas em tratamento, como o regime TDO, que é um método comprovadamente eficaz contra o abandono e responsável por impactos positivos no desfecho da TB. ${ }^{22}$

A implementação do TDO exige flexibilidade e organização por parte dos serviços, empoderamento da equipe quanto ao potencial desta estratégia a adesão e até mesmo inserção de tecnologias inovadoras, tal como chamada de vídeo para supervisão da ingesta medicamentosa. Entretanto, a sobrecarga de atividades, baixo número de profissionais e falta de qualificação e incentivo das equipes não contribuem para sua efetivação. ${ }^{23}$

Dessa forma, não somente a identificação de novos casos é uma importante ferramenta para o controle da TB, especialmente em PVHIV, mas também a garantia de continuidade do tratamento desta população, uma vez que o abandono favorece o desenvolvimento de TB-DR, eleva a complexidade dos casos e dificulta a cura. ${ }^{24}$ Para que isso se concretize, é necessário conhecer os fatores de risco para o abandono do tratamento 
ISSN 2179-6750

da população em questão e, a partir disso, definir e utilizar as estratégias adequadas para garantir a adesão terapêutica. ${ }^{25}$

Dado que a TB é um grande desafio nas PVHIV, devido à maior susceptibilidade de adoecimento nessa população, o rastreamento e diagnóstico precoce dos casos torna-se essencial para controle e não agravamento dos sinais e sintomas. A baciloscopia de escarro é um exame amplamente utilizado, capaz de detectar até $80 \%$ dos casos de TB pulmonar em adultos, sendo, portanto, indicada nos casos de suspeita clínica ou radiológica da doença. $^{2}$ Na população em questão, o teste foi realizado e com resultado negativo na maioria dos casos.

No entanto, apesar de sua sensibilidade, o exame apresenta baixa especificidade, ou seja, outros patógenos podem ser identificados através dele, retardando o diagnóstico. Para evitar esse problema, a recomendação é que se realize a cultura de escarro, teste altamente sensível e específico e, em seguida, o teste de sensibilidade, para avaliar a resistência do bacilo aos fármacos. ${ }^{2}$ Destaca-se ainda que, para o município de estudo, a cultura de escarro é recomendada para todos os casos de suspeita de TB, devido à importância epidemiológica das infecções por micobactérias não tuberculosa (MNT) no estado. Quanto ao teste de sensibilidade, a maioria dos casos estavam em branco/ignorado, o que prejudica no conhecimento se este exame foi solicitado, realizado e se o profissional teve acesso ao resultado durante o acompanhamento do caso.

Pode ser realizado, ainda, o TMR-TB, que tem sensibilidade superior à baciloscopia, alta especificidade, disponibilidade do resultado em 2 horas e detecta, ainda, se há resistência à rifampicina, sendo indicado para o diagnóstico de casos novos de TB pulmonar e laríngea. ${ }^{2}$ No presente estudo, este exame não foi realizado em $71 \%$ dos casos. Em parte, isso acontece porque o TMR-TB não é indicado para o diagnóstico de casos de retratamento, $^{2}$ que representam $32,9 \%$ dos casos de coinfecção da população em análise. No entanto, a baixa cobertura de realização do teste pode acarretar atraso no diagnóstico da TB, resultando em subnotificação de casos novos e dificultando o controle da doença.

De forma complementar aos exames laboratoriais, nos casos de TB pulmonar, pode ser realizada ainda a radiografia de tórax, a fim de observar padrões radiológicos sugestivos de TB, avaliar a extensão do acometimento e sua evolução durante o tratamento. ${ }^{2}$ A simplicidade e facilidade de realização desse tipo exame refletem nos resultados encontrados por este estudo, já que somente $7,6 \%$ dos casos não o realizaram.

Em relação aos exames de controle mensal, o preenchimento se deu como maioria em branco/ignorado, além de índices de não realização superiores a $20 \%$ em todos os meses de acompanhamento. Além disso, houve um usuário que obteve resultado da baciloscopia de escarro positivo até o sexto mês, tendo como possíveis causas a ineficácia do tratamento 
proposto, seja por resistência medicamentosa ou diagnóstico incorreto; deficiência no acompanhamento, uma vez que deveria ser investigada presença de outras comorbidades e realizados de exames complementares, tais como cultura de escarro e teste de sensibilidade; ou monitoramento da evolução através de exames inadequados para tal, como uso do TMR-TB ao invés da baciloscopia de escarro, levando a um resultado positivo independente da eficácia do tratamento, uma vez que se trata de um teste capaz de detectar fragmentos de DNA de bacilos. ${ }^{2}$

Destaca-se ainda que este mesmo caso foi encerrado com cura, o que leva ao questionamento deste desfecho, para qual a pessoa deve apresentar pelo menos duas baciloscopias negativas ao final do tratamento, ${ }^{2}$ apontando falha nas condutas adotadas e/ ou na retroalimentação dos dados.

Enquanto as unidades da Atenção Primária à Saúde - APS são responsáveis pelo rastreamento e tratamento da TB, monitorando para a eventual infecção pelo HIV através da oferta de teste rápido para o HIV tanto no momento do diagnóstico da TB quanto na suspeita clínica da doença, o SAE é o serviço responsável pelo acompanhamento da PVHIV, que, por se tratar de uma população suscetível ao desenvolvimento da TB, é responsável pelo rastreamento da doença nesta população, através de medidas como a investigação da TB em PVHIV anualmente e/ ou em caso de suspeita clínica de infecção pelo bacilo, sendo, portanto, nestes dois serviços onde preferencialmente deveriam ocorrer as notificações dos casos de coinfecção TB/HIV.

Entretanto, neste estudo, a elucidação diagnóstica da TB ocorreu, na maioria dos casos, no serviço de referência terciária, o que sugere a necessidade de internação diante da gravidade dos sinais e sintomas e reforça a fragilidade das ações de prevenção e rastreio da TB e/ou HIV na APS e SAE. Considerando um cenário onde essas condições não são trabalhadas quanto à sua prevenção, em que não há busca na comunidade dos portadores de ambas as doenças e que o serviço que acompanha o doente de TB não realiza testagem para o HIV e vice-versa, a pessoa pode desconhecer o seu diagnóstico, não buscar a devida assistência em tempo oportuno e, consequentemente, apresentar uma evolução clínica desfavorável, que leva a necessidade do indivíduo entrar no serviço de saúde através de um nível maior de complexidade de assistência e, portanto, a ser notificado diretamente no âmbito hospitalar.

Apesar da fragilidade apontada pelos locais de notificação, o tratamento da maioria dos casos de coinfecção TB/HIV analisados foi realizado no SAE, conforme preconizado pelo $\mathrm{MS}^{4}{ }^{4}$ mas que requer a continuidade da assistência e acompanhamento longitudinal do indivíduo, família e comunidade pela APS, uma vez que está se constitui como coordenadora do cuidado e ordenadora da Rede de Atenção à Saúde - RAS. ${ }^{26}$ Assim, ainda que o usuário 
ISSN 2179-6750

coinfectado seja tratado no SAE, a APS será responsável pela cogestão dos casos por meio do controle de contatos do caso índice, tratamento de infecção latente por TB (ILTB) ou TB ativa, quando necessário, realização do TDO e acompanhamento de todos os casos do seu território sanitário.

Os dados apontam para a necessidade de qualificação e sensibilização dos profissionais quanto à necessidade e importância do preenchimento e alimentação dos dados no sistema de forma adequada, uma vez que tais informações são indispensáveis para a avaliação dos casos e readequação da terapêutica, quando necessário, além de contribuir no planejamento das ações, monitoramento e intervenções necessárias para a vigilância e controle da TB. De modo complementar, destaca-se a importância de que estes profissionais sejam capacitados sobre os exames diagnósticos e de acompanhamento mensal da TB, a fim de compreender quando e quais devem ser solicitados, para que servem e qual a relevância de sua realização para o controle e cura da TB. A não realização do controle mensal reflete na fragilidade no vínculo entre usuários e serviço, e no acompanhamento e evolução dos casos, podendo resultar em terapêutica ineficaz e desfechos desfavoráveis e/ ou equivocados.

Outro problema observado neste estudo diz respeito ao controle de contatos. Identificou-se que a maioria dos casos notificados (74\%) possuía entre 1 e 5 contatos, mas que menos de $10 \%$ desses foram examinados. O controle de contatos permite identificar precocemente os casos ativos da doença e a ILTB em pessoas que convivem no mesmo ambiente que um SR, iniciando o tratamento antes do agravamento dos sintomas e disseminação do bacilo, constituindo, portanto, uma importante ferramenta na vigilância e controle da TB.

Dessa forma, torna-se necessário a comunicação e articulação entre os serviços e profissionais de saúde, dado que o rastreamento e avaliação dos contatos é de responsabilidade da APS, ainda que o usuário em questão seja acompanhado no SAE, considerando a territorialização, bem como ações de promoção e prevenção, dentre elas rastrear precocemente os casos e interromper a cadeia de transmissão, como uma das atribuições da APS.

Magnabosco et al. ${ }^{27}$ aponta ainda para a necessidade de integração dos programas de controle da TB com aqueles voltados ao HIV, de forma a implementar ações que abranjam os indivíduos de forma integral, e não fragmentada, considerando os aspectos sociais, econômicos e de saúde da coinfecção nos usuários. Ao mesmo tempo em que, a maior e melhor articulação entre os profissionais e diferentes serviços, pode abranger ações de promoção, abordagem da família e comunidade para redução, até mesmo, do estigma. 


\section{Perfil de encerramento dos casos}

Quanto aos desfechos encontrados, o percentual de cura está abaixo da meta estabelecida pela Organização Mundial da Saúde que preconiza, no mínimo, 85\% de cura e, no máximo, $5 \%$ de abandono. ${ }^{4}$ Nos casos de coinfecção analisados pelo estudo, somente $42 \%$ obtiveram desfecho de cura para TB, dado inferior ao encontrado para cura nos casos de TB pulmonar sem presença conhecida de infecção por HIV, em 2018, na capital do estado $(67,4 \%)^{28}$, demonstrando maior dificuldade no manejo da TB em casos de coinfecção.

Ainda que os resultados não sejam satisfatórios, deve-se atentar para o fato de que, se atingir um alto percentual de cura no caso da TB como morbidade única é uma tarefa difícil, nos casos de coinfecção TB/HIV esse desafio se torna ainda maior, dado o fato do impacto negativo do HIV sobre a TB, e vice-versa, tem como consequências maior dificuldade na adesão e eficácia do tratamento, além do aumento da letalidade de ambas as doenças. ${ }^{29}$ No entanto, levando em consideração que a TB é curável e o HIV é tratável, a não cura da TB em mais da metade dos casos é um índice preocupante, que aponta para necessidade de maior articulação dos serviços para identificação precoce, efetividade na organização da rede, melhor comunicação entre serviços e profissionais de saúde nos diferentes níveis de atenção, cogestão dos casos, fortalecimento de vínculo com os pacientes e adequação do manejo clínico, vigilância e monitoramento constante dos casos.

Um fator interessante observado é que mais de $70 \%$ dos abandonos aconteceram depois do terceiro mês de tratamento, evidenciando fragilidade no vínculo com o usuário e na adesão ao tratamento. Dentre os fatores que estão relacionados com o abandono ressalta-se a deficiência das informações e orientações fornecidas ao usuário, onde o profissional desconsidera a participação deste na gestão e corresponsabilização do seu cuidado, bem como a autonomia, participação do processo de tomada de decisão e subestima a eficiência do diálogo para compreensão da terapêutica. ${ }^{30}$

Por se tratar de um tratamento complexo, é importante que a equipe esteja preparada para a realização de orientações de forma que o usuário compreenda seu problema de saúde, o método de tratamento e a importância de segui-lo até o fim, tornando-o protagonista e responsável pelo seu autocuidado. Além disso, o usuário deve ser acompanhado até o fim do tratamento, com consultas periódicas e realização de exames para avaliação da efetividade da terapêutica, em que a equipe deve realizar a busca dos faltosos, quando houver, garantindo e fortalecendo o vínculo do usuário com o serviço.

Dos óbitos documentados, somente $0,7 \%$ dos casos ocorreram por TB, e os demais $(6,2 \%)$ por outras causas que pode estar relacionado com a aids. No entanto, vale destacar que essa categoria enquadra os óbitos por causas não patológicas, como violências, morte 
autoprovocada e acidentes de trânsito, que constituem as principais causas externas de óbito na Região Norte do país. ${ }^{31}$

Atenta-se também para o fato de que, a não comunicação entre os sistemas de informação, tais como o Sistema de Informação sobre Mortalidade e SINAN, levam à subnotificação deste desfecho, ${ }^{32}$ ressaltando a importância da vigilância dos óbitos para conhecimento das circunstâncias determinantes do óbito, conforme preconizado desde 2017. ${ }^{33}$

\section{Limitações do estudo}

Dentre as limitações do estudo, encontra-se a possível subnotificação dos casos de coinfecção TB/HIV, que torna invisível uma parcela da população atingida pelas patologias estudadas, podendo enviesar a análise e distanciar essas pessoas das decisões e planejamento das ações de saúde. Atenta-se ainda para a qualidade dos dados apresentados, considerando que a fragmentação do processo de trabalho para a disponibilização das informações exige capacitação profissional, para que estes entendam quais são e para que servem cada uma das variáveis contidas na ficha de notificação e como preencher completa e adequadamente cada uma delas, além do funcionamento e usabilidade do sistema, sensibilizando-os quanto à importância da coleta e processamento dos dados para a vigilância em saúde e como esta etapa contribui para o planejamento de ações direcionadas àquela população. Informações duplicadas, preenchidas erroneamente ou não preenchidas dificultam a visualização do real panorama da coinfecção no cenário estudado.

\section{Conclusão}

Este estudo permitiu a identificação do perfil sociodemográfico e clínico das pessoas com coinfecção TB/HIV na capital do estado de Rondônia, com predominância do sexo masculino, pardos, baixa escolaridade, com aids, alcoolismo e drogadição como principais agravos associados; PPL e PSR como principais populações vulneráveis, além de alinhamento entre o tipo de entrada (caso novo) e forma clínica (TB pulmonar) apontada por outras literaturas. No entanto, destacam-se percentuais preocupantes sobre a não realização de exames diagnósticos e de controle mensal da TB, bem como baixa realização de TDO e de avaliação de contatos, além de fragilidades importantes na execução das ações de manejo, vigilância e controle da doença e organização dos serviços no município para desenvolvimento de ações de prevenção, rastreio e 
identificação precoce, evidenciando necessidade de fortalecimento da cogestão dos casos de coinfecção TB/HIV pela APS.

Quanto aos desfechos, identificou-se baixa taxa de cura mesmo entre os usuários que cumpriram o tempo mínimo de tratamento e com percentual muito inferior quando comparado aos casos de TB pulmonar sem HIV no mesmo local de estudo; além do elevado percentual de abandono do tratamento, principalmente a partir do terceiro mês, evidenciando maior dificuldade no manejo e nas ações de controle da TB na população coinfectada TB/HIV no município em questão, bem como fragilidade no vínculo, corresponsabilização, implementação e efetivação de estratégias para busca de faltosos, monitoramento dos casos e incorporação de práticas de promoção da saúde e redução do estigma.

Os achados apontam para necessidade de melhora nas ações de prevenção de desenvolvimento da TB em PVHIV, diagnóstico e tratamento precoce de ambos os agravos, urgência no estabelecimento de fluxos assistenciais que favoreçam a articulação e integração da APS e SAE, dentre outros serviços que compõe a RAS. Evidencia-se, também, a necessidade de fortalecimento das ações de rastreamento da TB em PVHIV e do HIV nos indivíduos doentes de TB, de forma a contribuir para o diagnóstico e tratamento, em tempo hábil, para prevenção de ambas as doenças e recuperação da saúde dos indivíduos, através do preparo e qualificação dos profissionais de saúde quanto à realização e importância deste monitoramento e seu impacto no controle destes agravos, visando a promoção da qualidade de vida dos usuários.

Além disso, é imperativo maior sensibilização das equipes de saúde quanto ao preenchimento completo das fichas de notificação, retroalimentação e monitoramento dos dados no sistema de forma a contribuir para a visualização do panorama real da coinfecção TB/HIV, formulação de políticas de saúde, fortalecimento de estratégias exitosas e, consequentemente, diminuição dos índices de coinfecção TB/HIV e das várias questões e desafios relacionados a ela.

\section{Agradecimentos}

Ao Conselho Nacional de Desenvolvimento Científico e Tecnológico (CNPq), Programa Institucional de Bolsas de Iniciação Científica (PIBIC)/UNIR e ao Grupo de Estudos em Tuberculose (GET).

\section{Contribuição autoral}

ASM realizou a escolha do tema, análise e interpretação dos dados, redação, revisão 
crítica e aprovação do trabalho a ser publicado; GTM realizou interpretação, redação crítica e aprovação do trabalho a ser publicado; NHO realizou o planejamento, coleta e análise dos dados, orientação, supervisão, redação crítica e aprovação do trabalho a ser publicado.

\section{Referências}

1. World Health Organization. Global tuberculosis report 2019. Geneva: WHO; 2019.

2. Ministério da Saúde (BR). Manual de recomendações para o controle da tuberculose no Brasil. Brasília, DF: MS; 2019.

3. Ministério da Saúde (BR). Tuberculose. Bol Epidemiol. mar. 2020;(n. esp);1-39.

4. Ministério da Saúde (BR) Brasil livre da tuberculose: plano nacional pelo fim da tuberculose como problema de saúde pública. Brasília, DF: MS; 2017.

5. Santana S, Teixeira CFS, Rodrigues AS, Skalinski LM. Dificuldades, caminhos e potencialidades da descentralização do atendimento à tuberculose. J Health Biol Sci. 2020;8(1);1-5. http://dx.doi.org/10.12662/2317-3076jhbs.v8i1.2582.p1-5.2020.

6. Silva DR, Muñoz-Torrico M, Duarte R, Galvão T, Bonini EH, Arbex MA, et al. Fatores de risco para tuberculose: diabetes, tabagismo, álcool e uso de outras drogas. J Bras Pneumol. 2018;44(2):145-52. https://doi.org/10.1590/S1806-37562017000000443.

7. Instituto Brasileiro de Geografia e Estatística. População [Internet]. [Brasília, DF]: IBGE; c2017 [citado 29 maio 2020]. Disponível em: https://cidades.ibge.gov.br/brasil/ro/portovelho/panorama

8. Ministério da Saúde (BR). Cadastro nacional de estabelecimentos de saúde [Internet]. [Brasília, DF]: CNES; 2020 [citado 29 maio 2020]. Disponível em: http://cnes.datasus.gov.br/

9. Oliveira LB, Costa CRB, Queiroz AAFLN, Araújo TME, Sousa KAA, Reis RK. Análise epidemiológica da coinfecção tuberculose/HIV. Cogitare Enferm. 2018;23(1):e51016. http://dx.doi.org/10.5380/ce.v23i1.51016.

10. Bastos SH, Taminato M, Fernandes H, Figueiredo TMRM, Nichiata LYI, Hino P. Perfil sociodemográfico e de saúde da coinfecção tuberculose/HIV no Brasil: revisão sistemática. Rev Bras Enferm. 2019;72(5):1389-96. https://doi.org/10.1590/0034-71672018-0285. 
11. Marques CC, Medeiros ER, Sousa MES, Maia MR, Silva RAR, Feijão AR, et al. Casos de tuberculosis coinfectados por VIH en el estado del noreste brasileño. Enferm Actual Costa Rica. 2019;(36):62-76. http://dx.doi.org/10.15517/revenf.v0i36.33583.

12. Santos AC, Aquino RCA. Perfil epidemiológico de pacientes imunocomprometidos com tuberculose. Rev Eletron Estácio Recife. 2019;5(1):1-14.

13. Batista AT, Saldanha AAW, Furtado FMF. Auto conceito masculino e auto cuidado em saúde. Psicol Saude Doencas. 2017;18(3):859-69. http://dx.doi.org/10.15309/17psd180318.

14. Instituto Brasileiro de Geografia e Estatística. Pesquisa nacional por amostra de domicílios. Rio de Janeiro; IBGE; 2016.

15. Martins NFF, Abreu DPG, Silva BT, Semedo DSRC, Pelzer MT, Ienczak FS. Functional health literacy and adherence to the medication in older adults: integrative review. Rev Bras Enferm. 2017;70(4):868-74. https://doi.org/10.1590/0034-7167-2016-0625.

16. Carvalho Filho AX, Silva JP. Tuberculose em Minas Gerais e determinantes sociais. Rev Atenas Higeia. 2019;1(2):24-7.

17. Dooley KE, Chaisson RE. Tuberculosis and diabetes mellitus: convergence of two epidemics. Lancet Infect Dis. 209;9(12):737-46. https://doi.org/10.1016/S14733099(09)70282-8.

18. Dolan K, Wirtz A, Moazen B, Ndeffo-Mbah M, Galvani A, Kinner SA. Global burden of HIV, viral hepatitis, and tuberculosis in prisoners and detainees. Lancet. $2016 \mathrm{Sep}$ 10;388(10049):1089-1102. https://doi.org/10.1016/S0140-6736(16)30466-4.

19. Ferreira MR, Bonfim RO, Siqueira TC, Andrade RLP, Monroe AA, Villa TC, et al. Tubereculosis in prison and aspects associated with the diagnosis site. J Infect Dev Ctries. 2019 Nov 30;13(11):968-77. https://doi.org/10.3855/jidc.11522.

20. Rossetto M, Maffacciolli R, Rocha CMF, Oliveira DLLC, Serrant L. Coinfecção tuberculose/HIV/aids em Porto Alegre, RS: invisibilidade e silenciamento dos grupos mais afetados. Rev Gaucha Enferm. 2019;40:e2018003. https://doi.org/10.1590/19831447.2019.20180033.

21. Sharma SK, Mohan A. Extrapulmonary tuberculosis. Springer: Singapore; 2019. 
22. Silva ANC, Ross JR. Tratamento diretamente observado na tuberculose: imergindo em publicações científicas. J Manag Prim Health Care. 2020;12:1-11. https://doi.org/10.14295/jmphc.v12.303.

23. Cecilio HPM, Marcon SS. O tratamento diretamente observado da tuberculose na opinião de profissionais de saúde. Rev Enferm UERJ. 2016;24(1):e8425. https://doi.org/10.12957/reuerj.2016.8425.

24. Kibret KT, Moges Y, Memiah P, Biadgilign S. Treatment outcomes for multidrug-resistant tuberculosis under DOTS-Plus: a systematic review and meta-analysis of published studies. Infec Dis Poverty. 2017;6:7. https://doi.org/10.1186/s40249-016-0214-x.

25. Ferreira MRL, Bonfim RO, Siqueira TC, Orfão NH. Abandono do tratamento da tuberculose: uma revisão integrativa. Rev Enferm Contemp. 2018;7(1):63-71. https://doi.org/10.17267/2317-3378rec.v7i1.1579.

26. Mendes EV. As redes de atenção à saúde. 2a ed. Brasília, DF: Organização Panamericana de Saúde; 2015.

27. Magnabosco GT, Andrade RLP, Arakawa T, Monroe AA, Villa TCS. Desfechos dos casos de tuberculose em pessoas com HIV: subsídios para intervenção. Acta Paul Enferm. 2019;32(5):553-63. https://doi.org/10.1590/1982-0194201900077.

28. Ministério da Saúde (BR). Brasil livre da tuberculose: evolução dos cenários epidemiológicos e operacionais da doença. Bol Epidemiol. mar. 2019;50(9):1-18.

29. Jamal LF, Moherdaui F. Tuberculose e infecção pelo HIV no Brasil: magnitude do problema e estratégias para o controle. Rev Saude Publica. 2007;41(Suppl 1):104-110. https://doi.org/10.1590/s0034-89102007000800014.

30. Oliveira JF, Antunes MBC. Abandono anunciado ao tratamento da tuberculose em uma Unidade de Saúde da Família do Recife - a perspectiva do usuário. Rev APS. 2012;15(1):4-13.

31. Ministério da Saúde (BR). Saúde Brasil 2018: uma análise da situação de saúde e das doenças e agravos crônicos: desafios e perspectivas. Brasília, DF: MS; 2019.

32. Siqueira TC, Bonfim RO, Ferreira MRL, Orfão NH. Mortalidade entre os portadores de tuberculose em Porto Velho (RO). Saude Pesqui. 2018;11(3):441-50. https://doi.org/10.17765/1983-1870.2018v11n3p441-450. 
33. Ministério da Saúde (BR). Protocolo para vigilância do óbito com menção de tuberculose nas causas de morte. Brasília, DF: MS; 2017.

Amanda Silveira Mariano | https://orcid.org/0000-0002-0791-0790 http://lattes.cnpq.br/9037760657020604

Gabriela Tavares Magnabosco | https://orcid.org/0000-0003-3318-6748 http://lattes.cnpq.br/1952084216497152

Nathalia Halax Orfão | https://orcid.org/0000-0002-8734-3393

http://lattes.cnpq.br/7568632544062305 